\title{
Applicability of NGGM near-real time simulations in flood detection
}

DOI: https://doi.org/10.1515/jogs-2019-0011

Received October 26, 2018; accepted June 28, 2019

\begin{abstract}
The GRACE mission has demonstrated a tremendous potential for observing mass changes in the Earth system from space for climate research and the observation of climate change. Future mission should on the one hand extend the already existing time series and also provide higher spatial and temporal resolution that is required to fulfil all needs placed on a future mission. To analyse the applicability of such a Next Generation Gravity Mission (NGGM) concept regarding hydrological applications, two GRACE-FO-type pairs in Bender formation are analysed. The numerical closed loop simulations with a realistic noise assumption are based on the short arc approach and make use of the Wiese approach, enabling a self-de-aliasing of high-frequency atmospheric and oceanic signals, and a NRT approach for a short latency.

Numerical simulations for future gravity mission concepts are based on geophysical models, representing the timevariable gravity field. First tests regarding the usability of the hydrology component contained in the Earth System Model (ESM) by the European Space Agency (ESA) for the analysis regarding a possible flood monitoring and detection showed a clear signal in a third of the analysed flood cases. Our analysis of selected cases found that detection of floods was clearly possible with the reconstructed AOHIS/HIS signal in $20 \%$ of the tested examples, while in $40 \%$ of the cases a peak was visible but not clearly recognisable.
\end{abstract}

Keywords: Flood detection, Future gravity mission, Nearreal time, Time variable gravity

\section{Introduction}

Since the year 2000, the mass transport processes of the Earth system are observed by dedicated gravity missions such as CHAMP (CHAllenging Minisatellite Payload) (Reig-

*Corresponding Author: A. F. Purkhauser: Technische Universitat Munchen München, Germany, E-mail: anna.purkhauser@tum.de J. A. Koch, R. Pail : Technische Universitat Munchen München, Germany

ӘOpen Access. (cc) BY bution alone 4.0 License. ber et al. 1999) and GRACE/GRACE-FO (Gravity Recovery And Climate Experiment (Follow-On)) (Tapley et al. 2004; Flechtner et al. 2017). The CHAMP satellite mission's main observing technique is a high-low satellite-tosatellite tracking (hl-sst) leading to a spatial resolution of 500 to $1000 \mathrm{~km}$ (Baur 2013). The GRACE concept is based on twin satellites flying in a LEO (low Earth orbit) with the main observation being the K-band microwave lowlow satellite-to satellite tracking (ll-sst) between the two satellites. GRACE-FO features an additional inter-satellite laser ranging interferometer as technology demonstrator (Sheard et al. 2012), establishing that a further improvement of the ranging accuracy down to a few nanometres is possible.

Based on this observation techniques the computation of temporal gravity fields with a resolution of 1 month (Tapley et al. 2004), 10 days (Bruinsma et al. 2010; Tapley et al. 2013) and even 1 day solutions (Kurtenbach et al. 2009; Mayer-Gürr et al. 2016), the latter using a Kalman filter, are possible, supporting the global and continuous analysis of atmosphere, ocean, hydrology, ice and solid Earth (AOHIS) for hydrological processes (Rodell et al. 2009; Tiwari et al. 2009), ice mass melting (Luthcke et al. 2013; Velicogna et al. 2014), sea level risk (Willis et al. 2010), atmospheric circulation (Forootan et al. 2014), changes of the solid Earth like earthquakes (Han et al. 2013), and their interaction.

Possible NGGMs will have to address the issue of an anisotropic error spectrum and resulting strong striping features due to observation geometry in combination with temporal aliasing effects (Seo et al. 2007), hampering more challenging user requirements in terms of spatial resolution, time resolution and latency. A NGGM constellation usually consists of two GRACE-like pairs in a polar and an inclined orbit, also called Bender-pair, to address these issues. In addition to the improved scientific analysis that would be possible with such a satellite constellation, timevariable gravity field products shall contribute to operational services and applications such as water management, coastal vulnerability monitoring and forecasting of floods and droughts (Pail et al. 2015).

To analyse the applicability of a NGGM constellation regarding the detection and possible future prediction of 
droughts and floods, 6 months of data based on a Bendertype NGGM constellation was simulated. Chapter 1 introduces shortly the simulation environment, the orbit parameters of the satellite constellation, the noise characteristics used and the post-processing. Chapter 2 describes the used data sources and details the processing of the gravity fields towards a time series for floods and droughts detection. Among the results, a comprehensive analysis of the simulation itself, the analysis of the content of the hydrological layer, the comparison of the $\mathrm{H}$ component to HIS and AOHIS are presented, the applicability of the reconstructed gravity fields for the proposed application is studied and open questions within the analysis are discussed (see Chapter 3), followed by a short conclusion and outlook in Chapter 4.

Within the paper the following definitions are used: A flood is the rise and overflow of a large amount of water beyond its normal limits and can happen depending on the overall situation within hours, while a drought is the prolonged shortage in the water supply and can only be detected in long-term monitoring. Their possible detection is limited by the fact that with gravity data only socalled gravimetric floods and droughts are visible, meaning events that are associated with a change of mass and a corresponding change in the temporal gravity field. In this paper we focus on flood events.

\section{Simulation}

\subsection{Orbit design}

While various studies have analysed the potential of a second pair (Bender et al. 2008; Wiese et al. 2011; Wiese et al. 2012), the study by Elsaka et al. (2014) has concluded that a Bender configuration consisting of a polar and an inclined pair gives the best gain in accuracy on a global average. The orbit design itself is in accordance with the findings of the ESA-funded study SC4MGV (Assessment of Satellite Constellations for Monitoring the Variations in Earth Gravity Field) (Iran Pour et al. 2015). The main finding being that there is a certain freedom to tailor the orbits to potential applications, because a multitude of different scenarios delivered very comparable results regarding achievable gravity field performance. The study also showed that the retrieved gravity models did not have constant quality over time. Therefore, in order to improve the situation the orbits selected in this study have the same drift rate to ensure optimal interleaving at all times. The analysed constellation consists of a near-polar pair similar to GRACE
Table 1. Orbit parameters for satellite constellations.

\begin{tabular}{lccc}
\hline $\begin{array}{l}\text { Satellite } \\
\text { pair }\end{array}$ & $\begin{array}{c}\text { Altitude } \\
{[\mathrm{km}]}\end{array}$ & $\begin{array}{c}\text { Inclination } \\
\text { [degree] }\end{array}$ & $\begin{array}{c}\text { Inter-satellite } \\
\text { distance [km] }\end{array}$ \\
\hline Near- & 340 & 89 & 100 \\
polar & 355 & 70 & 100 \\
Inclined & & & \\
\hline
\end{tabular}

and an inclined pair with an inclination of $70^{\circ}$, see also Table 1. The state vectors used for the orbit integration are from the ESA-funded ADDCON study (Additional Constellation \& Scientific Analysis of the Next Generation Gravity Mission Concept) (Purkhauser et al. 2018).

\subsection{Numerical simulator}

The simulation was processed by a numerical closed-loop simulator available at the Institute of Astronomical and Physical Geodesy (IAPG) (Daras et al. 2015; Daras 2016) using the short-arc approach (Schneider 1969) with a low-low satellite-to-satellite tracking (ll-sst) and high-low satelliteto-satellite tracking (hl-sst) component sampled at $5 \mathrm{sec}$ onds. Additionally, the NRT processing consisting of a combination of the Wiese approach (Wiese et al. 2011) and a sliding window averaging at normal equation level (Purkhauser and Pail 2019), is used. The Wiese approach co-estimates a low spatial resolution gravity field at a short time interval together with higher resolution gravity fields sampled at longer time intervals, allowing for a selfdealiasing and the stable processing of solutions of a few days combined with a daily gravity field solution. The sliding window averaging allows for an optimal latency of one day, due to the fact that the data of each day is processed as soon as all necessary data products like EOP (Earth orientation product), rapid GNSS orbits and clocks are available, while the data of the first day in the previous solution is excluded. Consequently, the data analysis is performed with redundancies on the daily solution level and features overlapping gravity solutions. Based on this processing scheme, a variety of different data sets are available. More details on the approach can be found in Purkhauser and Pail (2019).

Since a short time sampling was desired, gravity fields with a temporal resolution of three days and a spatial sampling of $\mathrm{d} / \mathrm{o} 30$ corresponding to a spatial resolution of approx. $670 \mathrm{~km}$ or $160.000 \mathrm{~km}^{2}$, accompanied by daily solutions with a spatial resolution of $\mathrm{d} / \mathrm{o} 15$ corresponding to a spatial resolution of approx. $1300 \mathrm{~km}$ or $1.700 .000 \mathrm{~km}^{2}$ were determined. The gravity fields were processed from $1^{\text {st }}$ of January till $30^{\text {th }}$ of June of the year 2002. 


\subsection{Data}

The simulation is based on the static gravity field GOCO05s model (Mayer-Gürr and the GOCO Team 2015) and ESA's ESM (Dobslaw et al. 2015), a synthetic model of the time variable gravity field of the Earth available in spherical harmonics up to degree and order (d/o) 180 from the years 1996 to 2005 at 6-hourly snapshots. The time variable gravity field consists of AOHIS which are computed as coupled geophysical models as well as gravity field changes due to solid Earth processes like continuous glacial isostatic adjustment (GIA) or a sudden earthquake with co-seismic and post-seismic signals. It is used as model for the time variable gravity field information in order to perform the simulation study and also to validate the simulated gravity fields.

The $\mathrm{H}$ component of the AOHIS includes a global model of all terrestrially stored water. The model is validated with satellite altimetry over surface water bodies and also GRACE.

A realistic de-aliasing model for high-frequency mass variability in atmosphere and ocean is also provided by the ESM in two separate components (Dobslaw et al.2016). The AO error (AOerr) model represents both large-scale and small-scale errors with zero mean and a stationary variance.

As ocean models either the GOT4.7 (Goddard Ocean Tide) tide model (Ray 1999) or the EOT08a (Empirical Ocean Tide) model (Savcenko and Bosch 2008) are used. Starting from the same state vectors but different force models the orbits and observations representing the "true" world as closely as possible and a reference world are propagated. The observations of the "true" world are additionally superimposed by noise time series according to the potential measuring system (see Table 2).

\subsection{Stochastic modelling}

In the context of the simulations the following two error sources were considered: the laser ranging instrument noise (see Eq. (1)) and the accelerometer noise (see Eq. (2) and (3)). The noise characteristics of the error sources are all frequency dependent $(f)$ and are approximated by analytical equations in terms of range rates

$$
d_{\text {range rates }}=2 \cdot 10^{-8} \cdot 2 \pi f \sqrt{\left(\frac{10^{-2} \mathrm{~Hz}}{f}\right)^{2}+1} \frac{m}{s \sqrt{\mathrm{Hz}}}
$$

$$
\begin{aligned}
& d_{\text {acc. } x}=d_{\text {acc. } z}=10^{-11} \\
& \sqrt{\left(\frac{10^{-3} H z}{f}\right)^{4} /\left(\left(\frac{10^{-5} H z}{f}\right)^{4}+1\right)+1+\left(\frac{f}{10^{-1} H z}\right)^{4}} \\
& \frac{m}{s^{2} \sqrt{H z}}
\end{aligned}
$$

with $x$ being the along-track, $y$ across-track and $z$ the quasi-radial component. Since the satellite is assumed to fly in drag-free mode, the biggest part of the nongravitational forces is compensated by a propulsion system consisting of ion thrusters.

The error assumptions of NGGMs were provided from the consultancy support of Thales Alenia Space Italia (TAS-I). In addition to the sensor noise, the NGGM simulations includes uncertainties in the ocean tide model (represented by the differences between two different ocean tide models), see Table 2. The impact of orbit errors is taken into account by propagating $1 \mathrm{~cm}$ of white noise onto the orbit positions.

\subsection{Post Processing}

To enhance the signal-to-noise ratio (SNR) of the NRT results in post-processing, a time variable decorrelation (VADER) filter was applied (Horvath et al. 2018). The main relation between filtered ( $\hat{x}_{\alpha}^{V A D E R}$, see Eq. (4)) and unfiltered $(\hat{x})$ spherical harmonics coefficients, is given by

$$
\hat{x}_{\alpha}^{V A D E R}=(N+\alpha M)^{-1} N \hat{x}=W_{a} \hat{x}
$$

with the corresponding normal equation matrix $N$, the inverse signal variance matrix $M$ and the scaling factor $\alpha$, for an adjustment of the filter strength. These three components form the filter matrix $W_{\alpha}$. In the case of the simulation the signal variance matrix can easily be computed from the known true signal, namely the ESA ESM AOHIS. This is a kind of best-case scenario, but in (Horvath et al. 2018) it was shown that the influence of the chosen signal variance model on the filter result is rather small.

\section{Data Analysis}

The following chapter describes the process of creating time series of flooded areas. For the information on floods the collection of the Dartmouth Flood Observatory (DFO) is used (Brakenridge et al. 2002). The collected data of 
Table 2. Force and noise models of the "true" and reference world used in the full-scale simulations.

\begin{tabular}{lcc}
\hline model & "true" world & reference world \\
\hline Static gravity field (GF) model & GOCO05s & GOCO05s \\
Time varying GF model & ESA AOHIS & - \\
Ocean tide model & EOT08a & GOT4.7 \\
Noise model & Laser interferometer noise & - \\
Noise model & Accelerometer noise & - \\
\hline
\end{tabular}

large floods spans from 1985 to the present and is derived from the news, governmental, instrumental and remote sensing sources. It is available as Excel and shapefile with each flood having the outline of the affected area in longitude and latitude, time span, affected countries and area in square kilometres.

\subsection{Input data}

The flood time series is determined for the following data products:

- The ESM's H component to check the signal content of the hydrology component and investigate which flood events are visible.

- The whole ESM signal, namely AOHIS, which contains the full time variable gravity information, downsampled.

- The ESM's HIS signal consisting of the hydrological signal as well as the ice and solid Earth information, also down-sampled.

- The AOHIS reconstructed by the numerical closedloop simulation as described in Chapter 1 as NRT solutions with a temporal resolution of 3 days. Additionally, the reconstructed AOHIS is VADER filtered $(\alpha=1000)$.

- The HIS signal, reconstructed by subtracting the AO dealiasing product from the full reconstructed AOHIS.

To make the different data sets comparable, a downsampling of the ESM by computing a weighted mean of the $6 \mathrm{~h}$ snapshots to the temporal and spatial resolution of reconstructed signals (3 days, d/o 30-50) was necessary.

Additionally, an average year time series to enable the analysis of the deviation from the normal year is computed for each input signal. The computation is done from the ESA ESM model solely, which is available for 12 years (for further discussion see Section 3.4).

\subsection{Time series of specific areas}

The following process was applied for all data specified in Section 2.1:

- For a spatial representation in equivalent water heights (EWH, see Eq. (5)) (Wahr 1998) the spherical harmonics are evaluated on a grid with a spacing of $0.25^{\circ}$ :

$$
\begin{aligned}
E W H(\lambda, \theta)= & \frac{a \rho_{e}}{3 \rho_{w}} \sum_{n=0}^{\infty} \frac{2 n+1}{1+k_{n}} \sum_{m=0}^{n} \bar{P}_{n m}(\cos \theta) \\
& \left(\bar{C}_{n m} \cos m \lambda+\bar{S}_{n m} \sin m \lambda\right),
\end{aligned}
$$

where $\rho_{w}$ and $\rho_{e}$ represent the average density of water and Earth, $a$ the semi-major axis of the Earth, $k_{n}$ the love numbers and $c_{n m}$ and $s_{n m}$ represent the spherical harmonic (SH) coefficients

- Next, a definition of areas of interest is needed. This analysis is based on the flood data from the DFO. The flood data set includes the affected area itself in latitude and longitude coordinates. Additionally, circles situated at the centroid of the affected area with different radius are chosen. Also, river basins ${ }^{1}$ and subbasins $^{2}$ have been identified as potential areas of interest.

- These polygons were used to be intersected with the EWH information on the grid, keeping only the data points of the area of interest.

- A weighted average EWH ( $\bar{x}$, see Equ. 6) of the selected grid points is determined per epoch to form a time series for a specific area

$$
\begin{aligned}
& \bar{x}=\frac{\sum w x}{\sum w} \\
& w=\cos (\varphi)
\end{aligned}
$$

with $w$ the weight, depending on the latitude $\varphi$, and $x$ the EWH per grid point of the area of interest.

1 https://www.bafg.de/GRDC/EN/02_srvcs/22_gslrs/221_MRB/ riverbasins_node.html

2 ftp://rockyftp.cr.usgs.gov/vdelivery/Datasets/Staged/

Hydrography/WBD/National/GDB/ 
- This leads then to three identically processed time series of a specific area: One for the average year, the second containing the noise-free reference time-variable gravity field (thereafter called AOHIS, HIS or H); and third the reconstructed AOHIS or HIS as output of the numerical simulation.

\section{Results}

The following chapter will first validate the simulation results of the gravity field retrieval spatially as well as in the frequency domain. Further, the recoverability of the signal is also checked within the time series. Then the possibility to detect floods in comparison with an average year, computed from the same data basis (ESA ESM) is validated and open questions regarding the computation of the average year, definition of the area of interest and spatial leakage of flood events are discussed.

\subsection{Validation of the Simulation}

Within the simulation time period 179 3-day solution were computed. As Fig. 1 shows the solutions are stable and on average the reconstruction error surpasses the signal strength around degree 30 . To improve the performance of the gravity field solution, different settings for a VADER filter were tested and finally set to $\alpha=1000$. This allows for an improvement of the resolvability of on average up to $d / o$ 50. For the following analysis the spherical harmonics of the filtered gravity fields are truncated to $\mathrm{d} / \mathrm{o} 40$. The cumulative error in EWH for the non-filtered data till d/o 40 is $4.8 \mathrm{~cm}$, while the filtered cumulative error is reduced to $1.8 \mathrm{~cm} \mathrm{EWH}$.

\subsection{Signal Content of the ESA-ESM H component}

The signal content of the hydrology component of the ESAESM model was checked against the recorded floods by the DFO. For each of the 113 floods in the first half of the year 2002, a flood time series for the affected area was determined for both the $\mathrm{H}$ component directly as well as the average year computed from the same component with a temporal resolution of a day and spatial resolution of $\mathrm{d} / \mathrm{o}$ 100. After the exclusion of very small and therefore local floods (34\% of the data set) and the visual analysis of the remaining events of 74 floods a set of 15 flood cases (see Ta- ble 3) with clear visual indications, meaning an ascending trend and/or a peak in the flood time series at the indicated time period, was selected for further testing, marked with black circles in Fig. 2.

Next, the detected floods were examined regarding their signal amplitude in different $\mathrm{SH}$ resolutions. Figure 3 visualizes the flood time series for three examples for the hydrological component: On the top a smaller flood in Australia (a) with an affected area of approx. $60.000 \mathrm{~km}^{2}$ and short duration is depicted. In magenta the EWH minus the average EWH, computed from the 12 years of available ESM data, in the same area taking spherical harmonics till $\mathrm{d} / \mathrm{o}$ 100 into account is depicted. In blue and green the time series with a lower $\mathrm{SH}$ resolution, namely $\mathrm{d} / \mathrm{o} 50$ and 30 is displayed. The comparably large signal bias is due to its small spatial expansion, but strong amplitude and was also observed in other small scale examples. The other examples are in the US (b) with a large area of approx. $280.000 \mathrm{~km}^{2}$ and a long duration, and a middle scale flood in China (c) with an expansion of approx. $130.000 \mathrm{~km}^{2}$ and an intermediate duration. Both time series show only a small deviation when using a lower SH resolution. In all analysed cases the flood is visible in all $\mathrm{SH}$ resolutions, which is important for the following analysis. Also, the size of the analysed area of interest as well as the magnitude of the signal plays a role in the detectability of floods.

Below each time series figure the general area of the flood is visualized spatially at the beginning and in the middle of the indicated flood. The SH resolution from left to right is: $d / o$ 100, 50 and 30. The reduction of SH resolution and therefore the signal content can be observed in the spatial pattern and magnitude. The flood in Illinois (US) is large enough to be easily recognizable in the spatial plot as well, however, the indicated spatial expansion indicates a larger affected area. Overall, the spatial plot is not as easily interpretable as the computed time series.

\subsection{Signal Content of the ESA-ESM H vs HIS vs AOHIS component}

The NGGM gravity field retrieval can be done for the whole AOHIS signal. This is one of the main advantages of a NGGM concept over a single-pair. For floods, and in the future also droughts, the hydrology component is of interest. The main contributing components over continents hampering the detection of floods is the atmosphere. The HIS components can be retrieved from AOHIS by removing atmosphere and ocean $\mathrm{AO}$ ) via $\mathrm{AO}$ dealising products, also available from the ESA ESM model. 

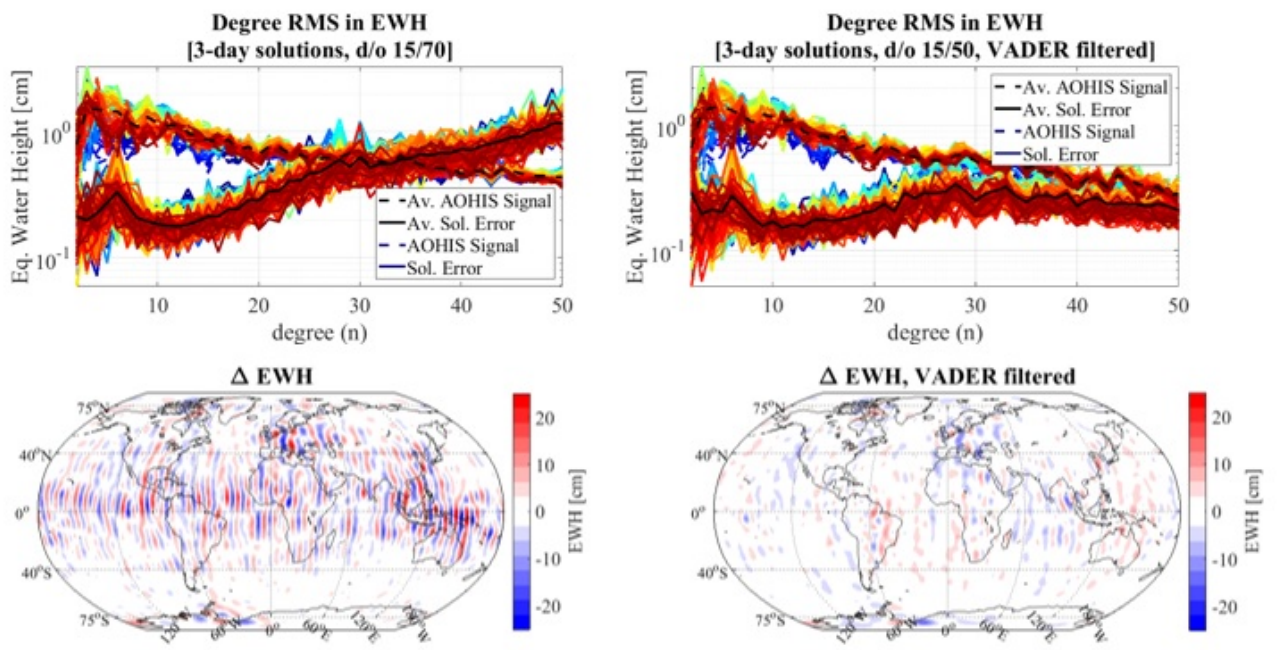

Fig. 1. Top: Degree RMS of the simulated EWH derived from the NGGM Bender pair without (left) and with (right) the VADER filter applied. All AOHIS signal as well as every error of the computed solutions is displayed in colours ranging from blue to red, with the average signal and error visualized in black. Bottom: Reconstructed AOHIS solution (first 3-day solution, d/o 50) minus the respective reference AOHIS signal without (left) and with (right) the VADER filter applied. The setting of the VADER filter is $\alpha=1000$.

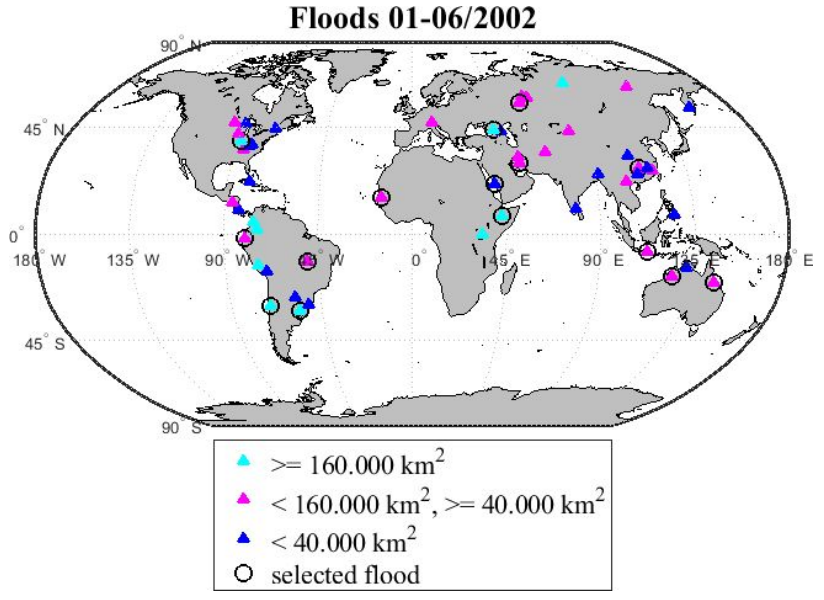

Fig. 2. Geographical locations of the floods in the time span of January to June 2002. According to the affected area, the floods are marked in cyan for floods equal or larger than $160.000 \mathrm{~km}^{2}$, magenta for floods equal or larger than $40.000 \mathrm{~km}^{2}$ and smaller than $160.000 \mathrm{~km}^{2}$. Small flood (smaller than $40.000 \mathrm{~km}^{2}$ ) are marked in blue. Floods smaller than $3.000 \mathrm{~km}^{2}$ are excluded from the graph. Floods selected for further analysis, due to a visibility of the indicated flood in the hydrological layer of the ESA ESM model are circled in black.

Figure 4 shows the EWH for the same three selected flood areas with the H, HIS and AOHIS component till $\mathrm{d} / \mathrm{o} 30$ in comparison. In grey the duration of the flood is marked. Clearly the most variations are visible in the full AOHIS signal visualized in red. These variations are due to the atmospheric component in the full signal. In comparison the HIS signal (displayed in cyan), without the atmosphere and ocean (not applicable in this case, due to analysing only areas on the continents) has clearly less fluctuation in the signal. However, this signal is only available after using AO-dealising products, which entail their own errors as well. And lastly in green the hydrology, which is the signal of interest.

Fig. 4 shows clearly the impact of the atmospheric signal in the AOHIS, as well as the potential of the HIS signal for the detection of floods. Note that both ice and solid Earth have smaller and also more long term characteristics compared to hydrology. The question is now how well the signal can be recovered in the closed-loop gravity field retrieval experiment to enable the monitoring and detection of floods.

\subsection{Flood detection from reconstructed signal}

The simulated scenario of a NGGM double pair mission allows for a full reconstruction of the AOHIS, due to the possibility of self-dealiasing with the Wiese approach. A first analysis of data is therefore the correlation of the reconstructed AOHIS to the ESA ESM AOHIS for the flooded zones. Table 4 shows the correlation of the reconstructed AOHIS and the original ESA ESM AOHIS in terms of percent and RMS error. Using the reconstructed signal directly till the SNR is met, leads on average to a correlation of $87.5 \%$, if the affected area is used as area of interest directly. The best cases indicate correlations of up to $99 \%$, while the 

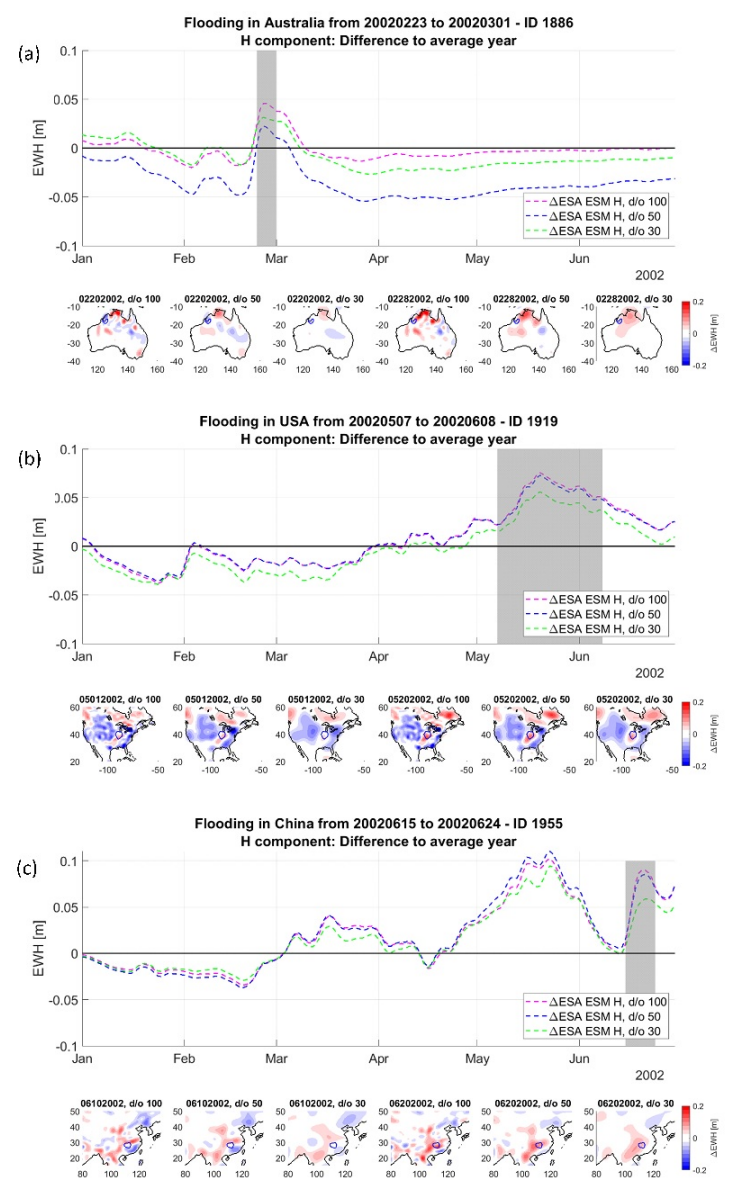

Fig. 3. The hydrology component of the ESA ESM displayed till d/o 100 (magenta), 50 (blue) and 30 (green) for the flood in Australia (a, ID 1886 according to the DFO), the US (b, ID 1919) and China (c, ID 1955). The duration of each flood is indicated with the grey shading. Below a spatial plot of the general affected area before and in the middle of the flood with $d / 0100,50$ and 30 is visualized. As area of interest the affected area according to the DFO is used, also called floodzone.

worst are about $78 \%$. These differences, however, cannot be directly linked to features like area, shape or location. The RMS values show value wise the same behaviour as the correlation in percent. The analysed case of Indonesia (ID 1870) is an outlier due to its shape and structure as an insular state with the biggest problem being the signal separation between land and ocean in coastal regions. Also, in case of floods close to the coast the generalized circular shape has to be considered with care or results dismissed, due to the possible inclusion of oceanic data in the averaged result.

Table 5 lists the correlation using the VADER filtered reconstructed signal. While the performance of the signal is expanded by 10 degrees, the correlation is on average decreased by $10 \%$. This reduction is caused due to the fil-

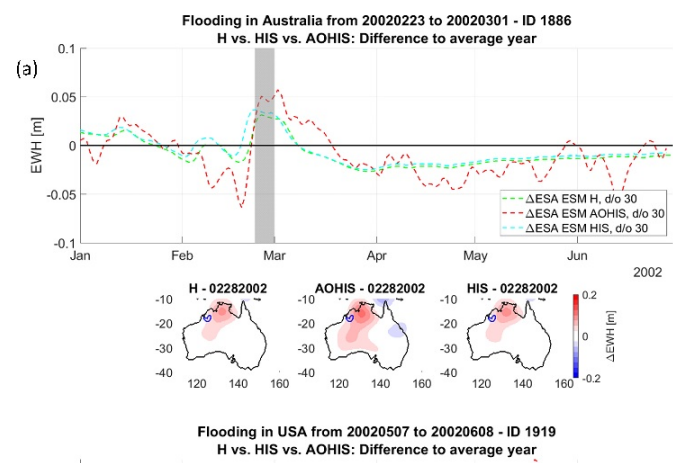

(b)
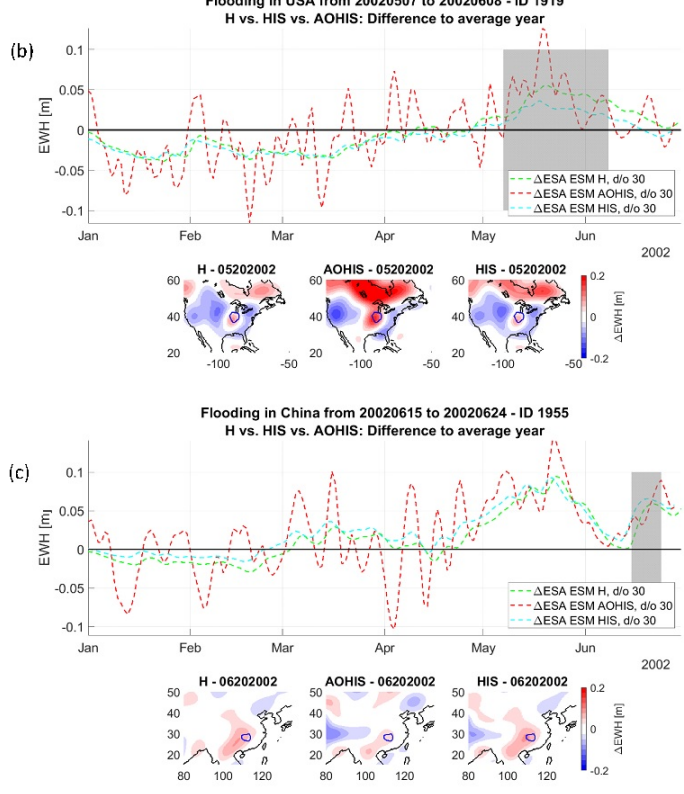

Fig. 4. Comparison of the H (green), HIS (cyan) and AOHIS (red) component of the ESA ESM for the chosen areas of interest. The variability of the whole AOHIS signal due to the atmosphere is clearly visible. The HIS signal is enhanced in the example of Australia (a), while in the example of the US (b) and China (c) it is dampened. The duration of the flood is indicated in grey. Below each time series graph, a spatial plot of the area for the H, AOHIS and HIS component is shown at a time within the flooding.

tering and inherent damping of the signal as well as spatial leakage due to the filtering process. Figure 5 shows the reconstructed AOHIS in the unfiltered version till $\mathrm{d} / \mathrm{o} 30$ and the VADER filtered reconstructed AOHIS signal till d/o 40. While the filter helps a lot with the typical GRACE striping, that in a NGGM concept is reduced but still visible, the computed time series displays the effects of the filtering as amplified peaks. Therefore, filtering for such an application has to be evaluated carefully, and based on the results of this analysis, its seams recommendable to filter the data on the flood time series level.

If a more generalized approach for the area of interest is chosen, in this case circles drawn around the centroids with different radius (for further discussion see Sec- 

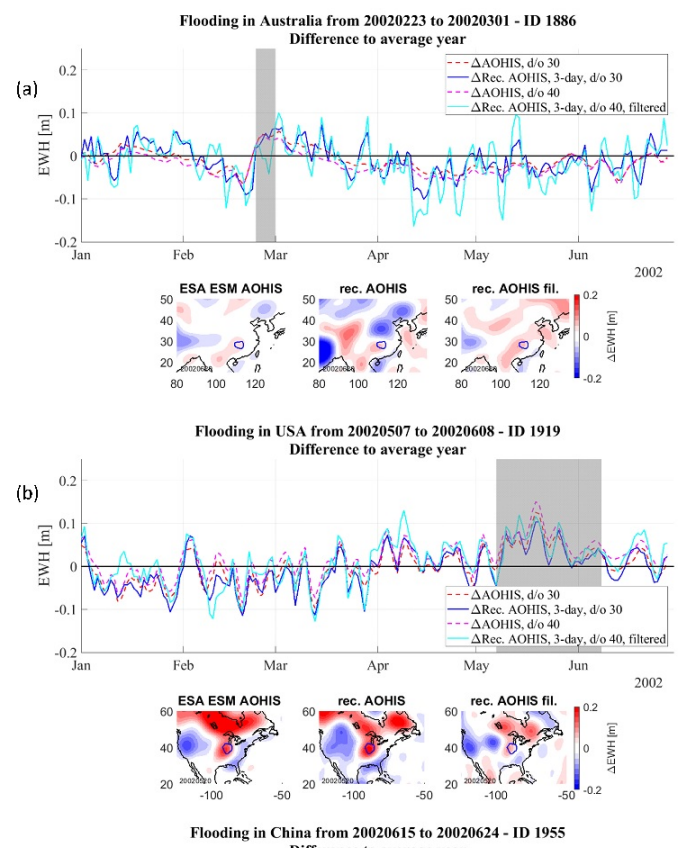

(c)

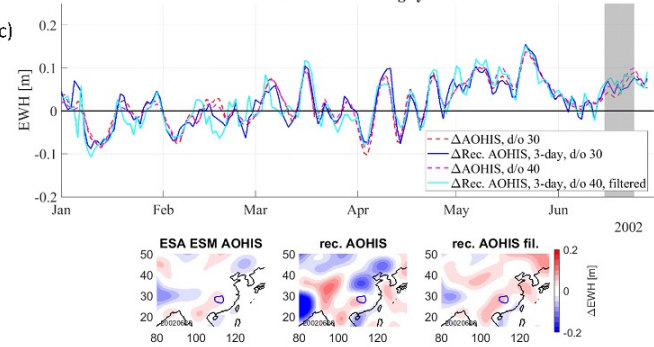

Fig. 5. Comparison between the ESA ESM AOHIS signal and the reconstructed AOHIS signal. The AOHIS signal is displayed for $\mathrm{d} / \mathrm{o}$ 30 in red, and for d/o 40 in magenta, while the reconstructed AOHIS signal with $\mathrm{d} / \mathrm{o} 30$ is visualized in blue and the VADER filtered rec. AOHIS signal with $d / 040$ is cyan. Below a spatial plot of the AOHIS reference signal, and the reconstructed AOHIS signal (filtered and not filtered) for $\mathrm{d} / \mathrm{o}$ is displayed.

tion 3.4), similar results can be achieved. A radius of $2^{\circ}$ means that the analysed area corresponds to the spatial resolution of a gravity retrieved with $\mathrm{d} / \mathrm{o} 50$, where on average $86.3 \%$ of the signal can be restored, while a larger area means a better recoverability by $89.3 \%$, which is even better than for the flooded area itself (see Table 4). A very similar result is also observable for the VADER filtered cases (see Table 5), with an improvement of the circle with a radius of $4^{\circ}$ due to the larger area used in the analysis.

Figure 6 displays the reconstructed AOHIS for the floodzone as well as the circular shape with a radius of 3 arc degree. The difference between the results is, as in the case of the correlation and RMS values, negligible.

Next, the atmosphere and ocean (AO) dealiasing component is subtracted from the full AOHIS signal to finally assess the reconstructed signal in comparison to the hy-

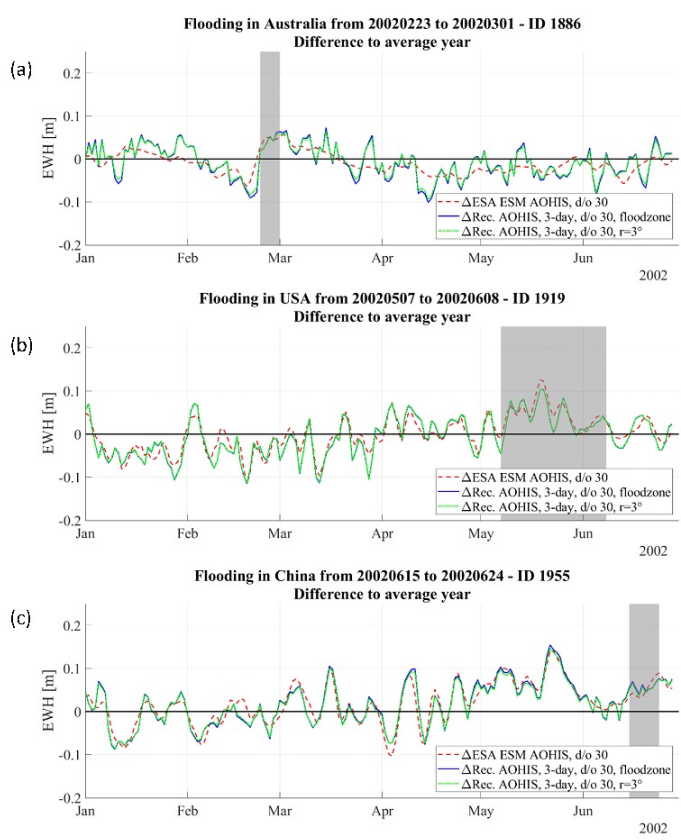

Fig. 6. The reconstructed AOHIS from the floodzone (blue) as well as the reconstructed AOHIS from a circular area of interest (green) with a radius of 3 arc degrees vs. the ESA ESM AOHIS (red). The choice of area of interest does not influence the resulting time series strongly. The time series of the flood in Australia in a coastal region shows more variation. However, since this flood is also the smallest one, it can be concluded, that there are no negative influences by the circular shape and the possible inclusion of oceanic signal.

drological input signal. Table 6 lists the correlation and RMS of the reconstructed HIS signal. While the correlation to the original time series is $20 \%$ less than when the full AOHIS is compared, the RMS stays in the same range. However, the change in correlation is not at the same level overall. Small floods in coastal areas experience the biggest degradation when using AO-dealiasing products to compute the reconstructed HIS signal. Also the second flood in Russia (ID 1962), with a large spatial expansion, but small amplitude, has a distinct decreased correlation factor.

Figure 7 visualizes the reconstructed HIS signal compared to the ESA ESM H component. It is clearly visible that the errors of the reconstructed gravity fields can be as large as the signal of interest. The reconstruction error compared to the signal amplitude depends on the size of the flooded area. While in the small example in Australia (Fig. 7a) the reconstruction error is dominating, the error becomes less important if larger areas are affected. Also the quality of the AO-dealiasing product plays a role.

To smoothen the time series a moving average with different window lengths is applied. Visually the moving average with the shortest window of 7 days performs best, 
while the moving average with the longest window of 31 days removes too much information from the data. The correlations in Table 7 comparing the reconstructed HIS to the desired $\mathrm{H}$ component of the ESA ESM reflects the results of Table 6, because the components ice and solid Earth do not produce a lot of valuable signal in the analysed regions. Also the results of the smoothed time series shows, that none of the applied moving averages performs optimal for the intended application.

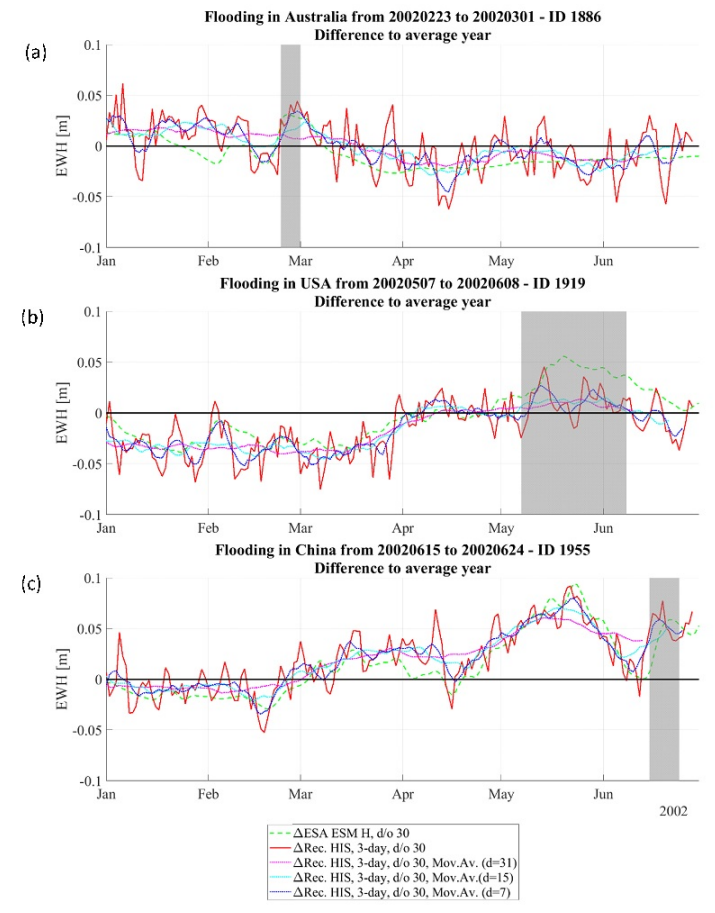

Fig. 7. The reconstructed HIS signal (by subtracting the AO Dealiasing products) for the 4 arc degree radius areas directly (red), with a moving average with $d=31$ (magenta), $d=15$ (cyan) and $d=7$ (blue) compared to the hydrological component of the ESA ESM (green).

From the 15 analysed flood events $20 \%$ are visually clearly identifiable as peaks in the reconstructed HIS signal, while $40 \%$ are visible, but are surrounded by other peaks and not as single flood event distinguishable. This result suggests, that the methodology is applicable for the suggested monitoring and detection of floods but needs improvement. The analysis showed clearly that a direct use of the reconstructed NGGM gravity fields without any post-processing is possible. The retrieval error, which depends on the size of the studied area, is still a big factor hampering the application based solely on gravity data. Also issues like average year (for more information see Section 3.4) and signal dealiasing with AO-dealiasing prod- ucts have to be addressed, before an actual implementation with unambiguous results can be undertaken.

\subsection{Open Questions}

\section{Average Year}

The computation of the reference year is done from the ESA ESM model solely, which is only available for 12 years. This means that the climatology expressed by the average for the reference year by itself is influenced by floods and droughts. A climatologically relevant time scale is at least 30 years and more, so that extreme years in terms of weather are balanced out by the other years of data. It can be expected that an average year of the recommended 30 or more years will be smoother and less likely to be influenced by onetime events such as floods and droughts themselves. A detailed comparison to the current averaged reference year (in spherical harmonics on a global level and as flood time series in EWH on a local level) has shown that for each analysed area, a different year (or even years) would have to be excluded to improve the average year significantly.

Since there are no plans for an extension of the ESAESM, a possibility to extend the time series would be to use GRACE data. However, different data sources would be mixed in that case. In a possible future automated process and alerting scheme the used average year is of great importance to give correct and precise information about the potential of a drought or flood.

\section{Analysed Area}

The analysed gravity time series initially was derived for the exact area of the flooding indicated by the DFO. While at this point the general detection of floods is analysed, in a future application the monitoring and detection beforehand is of interest. Therefore the next question is, which kind of definition of the area of interest would be best suitable for a more general approach.

In Fig. 8 different definitions of area of interest are visualized. The actually affected area (Fig. 8, top left) is only known after a flooding event took place. However, to analyse the potential of a monitoring system, and possibly detection system the affected area is an important factor. For an automated scheme circles on a grid could be an option to objectively judge each area - in Fig. 8 circles with the radius of $2^{\circ}, 3^{\circ}$ and $4^{\circ}$ are visualized. Overall these generally defined areas have given good results and are useful for such an analysis. Only the island state of Indonesia was not well covered by the analysis. Another possi- 


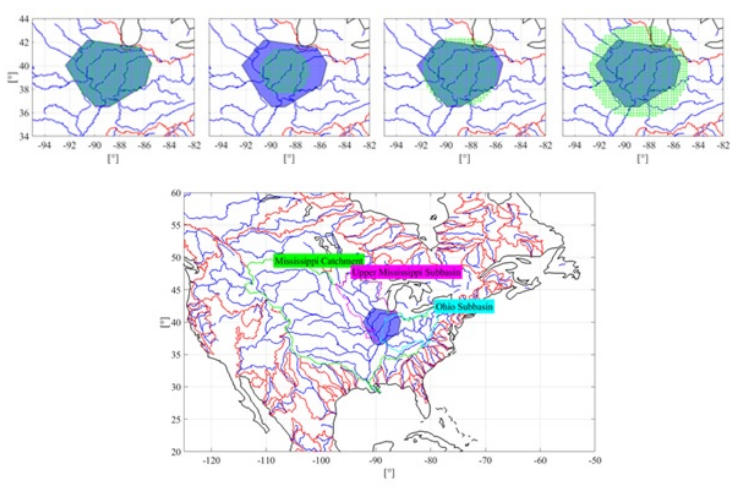

Fig. 8. Possible definitions of area of interest. Top: Affected area (historical data only), circles with $R=2,3$ and 4 arc degrees. Bottom: Catchment and Subbasins in the case of the US flooding (ID 1919).

bility are naturally defined areas or regions such as catchment borders and river basins. The selected example of the Mississippi catchment depicted in the bottom left of Fig. 8 demonstrates, that in a few cases the catchment is a too general selection since the area is too large. In this specific case a smaller-scale definition like the subbasin $U p$ per Mississippi River as depicted is more suitable, since not the whole river system was affected by the flood. However the overall analysis of the 15 cases showed, that only in a few cases, the catchments or the subbasin would have been a useful spatial definition.

\section{Signal Aliasing}

Another challenging aspect of the analysis is the occurrence of several flood events in a similar, neighbouring or overlapping region. Figure 9 depicts the time series of the Yangtze River catchment. The catchment is, as the Mississippi River catchment, rather large and consists of various rivers. Within the small time span of mid-May to the end of June, seven flooding's occurred in the region as the top right corner Fig. 9 depicts. The individual peaks are not distinguishable due to spatial leakage, with some peaks being visible in other time series as well, while others are not visible among the outliers. For such overlapping events additional data with a higher spatial resolution for a data assimilation is necessary to distinguish the events from each other.

\section{Post-Processing}

Both implemented post-processing strategies, namely the VADER filter as well as the moving average, did not achieve the hoped for results. Compared to other commonly used

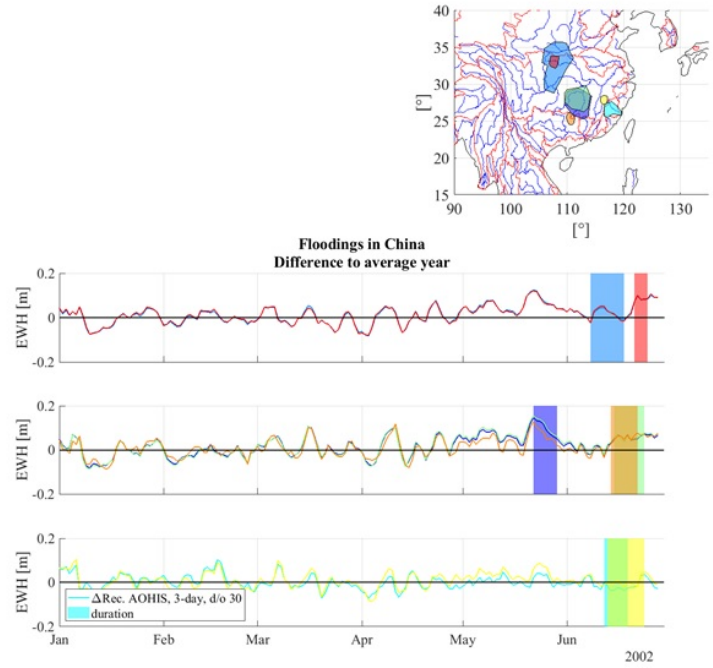

Fig. 9. Floods in China between January and June 2002 in a spatial plot (top, right) and the respective reconstructed HIS flood time series incl. ESA ESM HIS reference signal and the durations in the same colour.

filters, the VADER filter is specifically tailored to its data and signal. However, even with its fine-tuned filter matrix based on the solutions own NEQ, the filter still creates signal leakage on a spatial level. In comparison, the moving average window, indiscriminately smoothes the time series according to its input parameter and does not take into account the behaviour of the underlying time series. From this results it must be concluded further investigation is needed to give a conclusive answer on what postprocessing methodology achieves the best result for the application.

\section{Conclusion and Outlook}

The analysis within the paper is based on a closed loop simulation for a NGGM Bender pair using the Wiese approach for selfdealising and additionally a NRT retrieval approach to achieve a very short gravity field retrieval latency for the application of flood detection. For the analysed time span of the first half of the year 200215 floods, detectable in the hydrology component of ESA's ESM, are analysed based on the reconstructed HIS signal.

In $20 \%$ of the analysed floods a clear detection was possible with the simulated NGGM gravity fields only, while $40 \%$ were visible but not clearly distinguishable as flood due to other similar peaks in the time series. How well the flood is detectable is dependent on the flood characteristics itself like spatial expansion and signal magni- 
tude. When reconstructing HIS with the help of AO dealiasing products, the retrieval error of especially coastal and small floods suffer. However, a conclusive answer of the impact of different factors in the detectability could not be given within the scope of the paper. This would be a valid starting point for a more in-depth analysis.

When using different definitions of areas of interest similar result could be obtained, leading to the conclusion that a generalized approach would be a great option for a future service. The implementation of a post-processing scheme has shown, that although it is possible with NGGM to retrieve the complete AOHIS, a tailored post-processing is of essence to fully exploit the potential of NGGM constellations and their advantages. The implemented VADER filter as well as the moving average on time series level, are both not the optimal fit for the presented application.

Going forward several research topics have emerged from the presented analysis. On the one hand there are improvements to the data and analysis possible: First of all the average year has to be improved by adding additional years of data or removing outliers from the data set. One possibility would be to use real GRACE data to prolong the time series as long as no extension of the ESA-ESM is planned. Another, to calculate overall the average year not on a global, but on a local level, so that a potential outlier detection is meaningful.

Of interest in the future is also an analysis regarding droughts, which usually build up over years, or at least several months, so that at least a year-long simulation is necessary to quantify the possibilities in this area adequately.

Additionally, there are some general questions that still miss a conclusive answer: The influence of spatial leakage on neighbouring areas in regards of overlapping events. Also what kind of droughts and floods are detectable and in the end predictable via gravimetric satellite data (only).

\section{References}

Baur O (2013) Greenland mass variation from time-variable gravity in the absence of GRACE. Geophys. Res. Lett, Volume 40(16), 4289-4293. http://dx.doi.org/10.1002/grl.50881.

Bender PL, Wiese D and Nerem RS (2008) A Possible Dual-GRACE Mission with 90 Degree and 63 Degree Inclination Orbits. Proceedings of the 3rd International Symposium on Formation Flying, Missions and Technologies, ESA/ESTEC, Noordwijk, 23-25 April 2008, 1-6.

Brakenridge GR, Anderson E, and Caquard S (2002) Global Annual Maps of Affected Areas, Dartmouth
Flood Observatory, Hanover, USA, digital media, http://www.dartmouth.edu/\%7Efloods/Archives/index.html.

Bruinsma S, Lemoine J, Biancale R, and Valès N (2010)

CNES/GRGS 10-day gravity field models (release 2)

and their evaluation. Adv. Space Res. 45 (4), 587-601.

http://dx.doi.org/10.1016/j.asr.2009.10.012.

Daras I (2016) Gravity Field Processing Towards Future LL-SST Satellite Missions; Deutsche Geodätische Kommission der Bayerischen Akademie der Wissenschaften, Reihe C, Dissertationen, Heft 770, Verlag der Bayerischen Akademie der Wissenschaften, pp. 23-39. ISBN (Print) 978-3-7696-5182-9, ISSN 0065-5325, 2016.

Daras I, Pail R, Murböck M, and Yi W (2015) Gravity field processing with enhanced numerical precision for LL-SST missions. J. Geodesy 89 (2), 99-110. http://dx.doi.org/10.1007/s00190014-0764-2.

Dobslaw H, Bergmann-Wolf I, Dill R, Forootan E, Klemann V, Kusche J, and Sasgen I (2015) The updated ESA Earth System Model for future gravity mission simulation studies. Journal of Geodesy, Vol. 89, p. 505-513, https://doi.org/10.1007/s00190-014-07878

Dobslaw H, Bergmann-Wolf I, Forootan E, Dahle C, Mayer-Gürr T, Kusche J, and Flechtner F (2016) Modeling of present-day atmosphere and ocean non-tidal de-aliasing errors for future gravity mission simulations. Journal of Geodesy, 90, 5, pp. 423-436, https://doi.org/10.1007/s00190-015-0884-3

Elsaka B, Raimondo J-C, Brieden Ph, Reubelt T, Kusche J, Flechtner F, Iran Pour S, Sneeuw N, and Müller J (2014) Comparing Seven Candidate Mission Configurations for Temporal Gravity Retrieval through Full-Scale Numerical Simulation. Journal of Geodesy, 88, 31-43. http://dx.doi.org/10.1007/s00190-0130665-9.

Flechtner F, Webb F, and Watkins M (2017) Current Status of the GRACE Follow-On Mission. Geophysical Research Abstracts, vol. 19, EGU2017-4566, EGU General Assembly 2017. Deutsche Geodätische Kommission der Bayerischen Akademie der Wissenschaften, Reihe B, Angewandte Geodäsie, 318.

Forootan E, Didova O, Schumacher M, Kusche J, and Elsaka B (2014) Comparisons of atmospheric mass variations derived from ECMWF reanalysis and operational fields over 2003-2011, Jounral of Geodesy, 88, 503-514. http://doi.org/10.1007/s00190014-0696-x

Han S, Riva R, Sauber J, and Okal E (2013) Source parameter inversion for recent great earthquakes from a decade-long observation of global gravity fields, J. Geophys. Res. Solid Earth, 118, 1240-1267, http://dx.doi.org/10.1002/jgrb.50116.

Horvath A, Murböck M, Pail R, and Horwath M (2018) Decorrelation of GRACE time variable gravity field solutions using full covariance information. Geosciences Vol. 8(9), ISSN 2076-3263. https://doi.org/ 10.3390/geosciences 8090323

Iran Pour S, Reubelt T, Sneeuw N, Daras I, Murböck M, and Gruber $T$ (2015) Assessment of satellite constellations for monitoring the variations in earth gravity field - SC4MGV, ESA - ESTEC Contract No. AO/1-7317/12/NL/AF, Final Report.

Kurtenbach E, Mayer-Gürr T, and Eicker A (2009) Deriving daily snapshots of the Earth's gravity field from GRACE L1B data using Kalman filtering. Geophys. Res. Lett. 36 (17), L17102. http://dx.doi.org/10.1029/2009GL039564.

Luthcke SB, Sabaka T, Loomis B, Arendt A, McCarthy J, and Camp J (2013) Antarctica, Greenland, and Gulf of Alaska land-ice evolu- 
tion from an iterated GRACE global mascon solution, J. Glaciol., 59(216), 613-631. https://doi.org/doi:10.3189/2013jog12j147

Mayer-Gürr T and the GOCO Team (2015): The combined satellite gravity field model GOCO05s. Presentation at EGU 2015, Vienna, April 2015.

Mayer-Gürr T, Jäggi A, Meyer U, Yoomin J, Susnik A, Weigelt M, van Dam T, Flechtner F, Gruber C, Günter A, Gouweleeuw B, Kvas A, Klinger B, Flury J, Bruinsma J, Lemoine JM, Zwenzner H, Bourgogne S, and Bandikova T (2016) European Gravity Service for Improved Emergency Management - Status and Project Highlights. Geophysical Research Abstracts, vol. 18, EGU201614970, EGU General Assembly 2016.

Pail R, Bingham R, Braitenberg C, Dobsalw H, Eicker A, and Güntner A (2015) Science and User Needs for Observing Global Mass Transport to Understand Global Change and to Benefit Society. Surveys in Geophysics 36 (743). https://doi.org/10.1007/s10712-015-9348-9.

Purkhauser AF and Pail R (2019) Near-real time gravity field retrieval on short time scales in the context of next generation gravity missions: Geophysical Journal International, ggz084, https://doi.org/10.1093/gji/ggz084

Purkhauser AF, Pail R, Hauk M, Visser P, Sneeuw N, and Saemian P (2018) Gravity Field Retrieval of Next Generation Gravity Missions regarding Geophysical Services: Results of the ESA-ADDCON Project. European Geosciences Union General Assembly 2018. https://meetingorganizer.copernicus.org/EGU2018/EGU20182770.pdf.

Ray RD (1999) A global ocean tide model from topex/Poseidon altimetry: Got99.2, Tech. rep., NASA Technical Memorandum 209478.

Reigber C, Schwintzer P, and Lühr H (1999) The CHAMP geopotential mission, in Bollettino di Geofisica Teoretica ed Applicata, 40/3-4, September-December 1999, Proceedings of the Second Joint Meeting of the International Gravity and the International Geoid Commission, Trieste 1998 September 7-12, ISSN 0006-6729, pp. 285-289, eds Marson I, \& Sünkel H.

Rodell M, Velicogna I, and Famiglietti J (2009) Satellite-based estimates of groundwater depletion in India, Nature, 460, 9991002. https://doi.org/10.1038/nature08238

Savcenko R and Bosch W (2008) EOT08a - Empirical Ocean Tide Model from Multi-Mission Satellite Altimetry. DGFI Report, München, Germany, 81.

Schneider M (1969) Outline of a general orbit determination method, in Space Research IX, Proceedings of Open Meetings of Working Groups (OMWG) on Physical Sciences of the $11^{\text {th }}$ Plenary Meeting of the Committee on Space Research (COSPAR), Tokyo, edited by K.S.W. Champion, P.A. Smith, and R.L. Smith-Rose, pp. 37-40, North Holland Publ. Company, Mitteilungen aus dem Institut für Astronomische und Physikalische Geodäsie, Nr. 51., Tokyo, Japan.

Seo K-W, Wilson CR, Chen J, and Waliser D (2007) GRACE's spatial aliasing error. Geophysical Journal. https://doi.org/10.1111/j.1365-246X.2007.03611.x.

Sheard BS, Heinzel G, Danzmann K, Shaddock D, Kilpstein W, Folkner W (2012) Intersatelite laser ranging instrument for the GRACE Follow-On mission. J. Geodesy 86 (12), 1083-1095. http://dx.doi.org/10.1007/s00190-012-0566-3.

Tapley BD, Bettadpur S, Watkins M, and Reigber C (2004) The gravity recovery and climate experiment experiment, mission overview and early results, Geophys. Res. Lett., 31, L09607, http://dx.doi.org/10.1029/2004GL019920.

Tapley BD, Flechtner F, Bettadpur S, and Watkins M (2013) The Status and Future Prospect for GRACE After the First Decade. American Geophysical Union, Fall Meeting 2013, abstract \#G32A01.2013AGUFM.G32A.01T.

Tiwari VM, Wahr J, and Swenson S (2009) Dwindling groundwater resources in northern India, from satellite gravity observations. Geophys. Res. Lett. 36, L18401. http://dx.doi.org/10.1029/2009GL039401.

Velicogna I, Sutterley TC, and van den Broeke MR (2014) Regional acceleration in ice mass loss from Greenland and Antarctica using GRACE time-variable gravity data. Geophys. Res. Lett. 41 (22), 8130-8137. http://dx.doi.org/10.1002/2014/GL061052.

Wahr J, Molenaar M, and Bryan F (1998), Time variability of the earth's gravity field: Hydrological and oceanic effects and their possible detection using grace, Journal of Geophysical Research: Solid Earth, 103 (B12), 30,205.

Wiese D, Nerem R, and Han S-C (2011) Expected Improvements in Determining Continental Hydrology, Ice Mass Variations, Ocean Bottom Pressure Signals, and Earthquakes Using Two Pairs of Dedicated Satellites for Temporal Gravity Recovery. Journal of Geophysical Research, 116, 405. http://dx.doi.org/10.1029/2011JB008375.

Wiese D, Nerem R, and Lemoine F (2012) Design considerations for a dedicated gravity recovery satellite mission consisting of two pairs of satellites. Journal of Geodesy 86:81-98. http://dx.doi.org/10.1007/s00190-011-0493-8.

Wiese DN, Visser P, and Nerem RS (2011) Estimating low resolution gravity fields at short time intervals to reduce temporal aliasing errors. Adv. Space Res. 48 (6), 1094-1107. http://dx.doi.org/10.1016/j.asr.2011.05.027

Willis JK, Chambers DP, and Kuo CY (2010) Global sea level rise: recent progress and challenges for the decade to come. Oceanography 23, 26-35. http://dx.doi.org/10.5670/oceanog.2010.03. 


\section{Tables}

Table 3. Analysed floods selected from the DFO archive. The ID $\left(^{\star}\right)$ is taken from the archive.

\begin{tabular}{|c|c|c|c|c|c|c|}
\hline ID* & $\begin{array}{l}\text { Country } \\
\text { [main] }\end{array}$ & $\begin{array}{l}\text { Area } \\
{\left[\mathrm{km}^{2}\right]}\end{array}$ & Coast/Inland & Shape & $\begin{array}{c}\text { Beginning } \\
\text { [dd.mm.yyyy] }\end{array}$ & $\begin{array}{c}\text { Duration } \\
\text { [days] }\end{array}$ \\
\hline 1863 & Iran & 59660 & Coast/Inland & L-shaped & 11.01 .2002 & 3 \\
\hline 1866 & Senegal & 62710 & Coast/Inland & Rectangular & 09.01 .2002 & 4 \\
\hline 1870 & Indonesia & 41000 & Various ilands & Circles & 27.01.2002 & 17 \\
\hline 1881 & Australia & 47740 & Coast/Inland & S-shaped & 15.02 .2002 & 4 \\
\hline 1885 & Brazil & 139100 & Inland & Elongated & 15.01 .2002 & 78 \\
\hline 1886 & Australia & 57870 & Coast/Inland & L-shaped & 23.02 .2002 & 7 \\
\hline 1890 & Ecuador & 52930 & Coast & Rectangular & 06.03 .2002 & 55 \\
\hline 1902 & Saudi Arabia & 22810 & Coast & Rectangular & 08.04 .2002 & 6 \\
\hline 1907 & Ethiopia & 282500 & Inland & Square & 16.04 .2002 & 6 \\
\hline 1919 & USA & 286800 & Inland & Round & 07.05 .2002 & 33 \\
\hline 1932 & Chile & 166900 & Coast & Elongated & 24.05 .2002 & 13 \\
\hline 1939 & Uruguay & 205300 & Coast/Inland & Square & 23.04 .2002 & 17 \\
\hline 1946 & Russia & 66440 & Inland & Round & 09.06 .2002 & 7 \\
\hline 1955 & China & 130300 & Inland & Round & 15.06 .2002 & 10 \\
\hline 1962 & Russia & 224600 & Inland & Rectangular & 19.06 .2002 & 13 \\
\hline
\end{tabular}


Table 4. Correlation [\%] and RMS [cm] of reconstructed AOHIS signal to the original ESA ESM AOHIS for the 15 selected study cases (d/o 30 ).

\begin{tabular}{|c|c|c|c|c|c|c|c|c|}
\hline \multirow[t]{3}{*}{ ID } & \multicolumn{8}{|c|}{ Reconstructed AOHIS, d/o 30 [\%] } \\
\hline & \multicolumn{2}{|c|}{ Floodzone } & \multicolumn{2}{|c|}{ Circle, $R=2^{\circ}$} & \multicolumn{2}{|c|}{ Circle, $R=3^{\circ}$} & \multicolumn{2}{|c|}{ Circle, $R=4^{\circ}$} \\
\hline & Corr [\%] & RMS [cm] & Corr [\%] & RMS $[\mathrm{cm}]$ & Corr $[\%]$ & RMS $[\mathrm{cm}]$ & Corr [\%] & $\mathrm{RMS}[\mathrm{cm}]$ \\
\hline 1863 & 83.5 & 1.8 & 81.3 & 2.0 & 84.1 & 1.8 & 87.4 & 1.6 \\
\hline 1866 & 85.2 & 1.8 & 85.6 & 1.8 & 87.4 & 1.6 & 89.6 & 1.3 \\
\hline 1870 & 79.3 & 1.2 & 66.5 & 2.3 & 69.1 & 2.0 & 73.2 & 1.7 \\
\hline 1881 & 82.0 & 2.1 & 81.2 & 2.1 & 83.5 & 1.9 & 86.5 & 1.7 \\
\hline 1885 & 95.7 & 2.3 & 96.0 & 2.3 & 96.3 & 2.1 & 96.8 & 1.8 \\
\hline 1886 & 83.5 & 2.9 & 83.7 & 2.8 & 85.3 & 2.6 & 87.5 & 2.2 \\
\hline 1890 & 90.8 & 2.3 & 91.5 & 2.2 & 92.3 & 1.9 & 93.5 & 1.6 \\
\hline 1902 & 80.4 & 1.9 & 82.3 & 1.8 & 85.0 & 1.6 & 88.2 & 1.3 \\
\hline 1907 & 78.1 & 1.8 & 75.3 & 2.2 & 77.8 & 1.9 & 81.2 & 1.6 \\
\hline 1919 & 90.1 & 1.9 & 89.2 & 2.1 & 89.9 & 1.9 & 90.5 & 1.8 \\
\hline 1932 & 78.9 & 1.7 & 79.2 & 1.8 & 79.1 & 1.6 & 79.1 & 1.5 \\
\hline 1939 & 98.1 & 1.9 & 98.1 & 2.0 & 98.1 & 1.9 & 98.2 & 1.7 \\
\hline 1946 & 99.3 & 0.7 & 99.3 & 0.7 & 99.3 & 0.6 & 99.4 & 0.6 \\
\hline 1955 & 93.6 & 1.9 & 93.4 & 1.9 & 93.6 & 1.8 & 94.0 & 1.6 \\
\hline 1962 & 94.7 & 1.0 & 92.6 & 1.3 & 93.1 & 1.2 & 93.9 & 1. \\
\hline & 87.5 & 1.8 & 86.3 & 2.0 & 87.6 & 1.8 & 89.3 & 1.5 \\
\hline
\end{tabular}


Table 5. Correlation [\%] and RMS [cm] of reconstructed and VADER filtered AOHIS signal to the original ESA ESM AOHIS for the 15 selected study cases (d/o 40).

\begin{tabular}{|c|c|c|c|c|c|c|c|c|}
\hline \multirow[t]{3}{*}{ ID } & \multicolumn{8}{|c|}{ Reconstructed AOHIS, VADER, d/o 40 [\%] } \\
\hline & \multicolumn{2}{|c|}{ Floodzone } & \multicolumn{2}{|c|}{ Circle, $R=2^{\circ}$} & \multicolumn{2}{|c|}{ Circle, $R=3^{\circ}$} & \multicolumn{2}{|c|}{ Circle, $R=4^{\circ}$} \\
\hline & Corr [\%] & RMS [cm] & Corr [\%] & RMS [cm] & Corr [\%] & RMS [cm] & Corr [\%] & RMS $[\mathrm{cm}]$ \\
\hline 1863 & 75.8 & 2.3 & 70.8 & 2.5 & 75.7 & 2.2 & 82.0 & 1.8 \\
\hline 1866 & 68.4 & 2.4 & 70.5 & 2.3 & 76.8 & 2.0 & 83.9 & 1.5 \\
\hline 1870 & 70.4 & 1.7 & 52.0 & 4.2 & 56.7 & 3.4 & 64.4 & 2.4 \\
\hline 1881 & 73.5 & 2.8 & 72.2 & 2.9 & 77.1 & 2.4 & 83.1 & 1.9 \\
\hline 1885 & 93.7 & 3.2 & 94.2 & 3.0 & 95.2 & 2.6 & 96.3 & 2.0 \\
\hline 1886 & 66.8 & 4.7 & 67.7 & 4.6 & 73.0 & 3.8 & 79.8 & 3.0 \\
\hline 1890 & 88.9 & 3.1 & 90.1 & 2.9 & 91.5 & 2.4 & 93.3 & 1.8 \\
\hline 1902 & 50.9 & 2.6 & 56.1 & 2.4 & 65.6 & 2.0 & 77.5 & 1.6 \\
\hline 1907 & 70.3 & 2.3 & 65.9 & 3.0 & 71.2 & 2.5 & 78.0 & 1.9 \\
\hline 1919 & 74.2 & 3.2 & 68.9 & 3.7 & 73.0 & 3.3 & 78.2 & 2.7 \\
\hline 1932 & 68.3 & 2.4 & 73.4 & 2.5 & 74.2 & 2.1 & 75.5 & 1.8 \\
\hline 1939 & 96.7 & 2.6 & 96.4 & 2.8 & 96.8 & 2.5 & 97.3 & 2.1 \\
\hline 1946 & 98.0 & 1.1 & 98.1 & 1.1 & 98.3 & 1.0 & 98.6 & 0.9 \\
\hline 1955 & 93.7 & 2.5 & 93.3 & 2.6 & 93.7 & 2.2 & 94.3 & 1.8 \\
\hline \multirow[t]{2}{*}{1962} & 92.9 & 1.3 & 86.0 & 1.9 & 87.8 & 1.7 & 90.1 & 1.4 \\
\hline & 78.8 & 2.5 & 77.0 & 2.8 & 80.4 & 2.4 & 84.8 & 1.9 \\
\hline
\end{tabular}


Table 6. Correlation [\%] and RMS [cm] of reconstructed HIS signal to the original ESA ESM HIS for the 15 selected study cases (d/o 30 ).

\begin{tabular}{|c|c|c|c|c|c|c|c|c|}
\hline \multirow[t]{3}{*}{ ID } & \multicolumn{8}{|c|}{ Reconstructed HIS (AO + Error) } \\
\hline & \multicolumn{2}{|c|}{ Floodzone } & \multicolumn{2}{|c|}{ Circle, $R=2^{\circ}$} & \multicolumn{2}{|c|}{ Circle, $R=3^{\circ}$} & \multicolumn{2}{|c|}{ Circle, $R=4^{\circ}$} \\
\hline & Corr [\%] & RMS [cm] & Corr [\%] & RMS [cm] & Corr [\%] & RMS $[\mathrm{cm}]$ & Corr [\%] & RMS [cm] \\
\hline 1863 & 31.6 & 2.0 & 30.2 & 2.1 & 29.6 & 2.0 & 28.6 & 1.8 \\
\hline 1866 & 63.1 & 1.9 & 63.9 & 1.9 & 67.4 & 1.7 & 71.7 & 1.4 \\
\hline 1870 & 46.9 & 1.3 & 20.9 & 2.4 & 24.7 & 2.1 & 31.4 & 1.8 \\
\hline 1881 & 63.9 & 2.2 & 62.6 & 2.2 & 64.4 & 2.0 & 67.1 & 1.7 \\
\hline 1885 & 96.0 & 2.4 & 96.3 & 2.3 & 96.6 & 2.1 & 97.0 & 1.8 \\
\hline 1886 & 63.3 & 2.9 & 63.0 & 2.8 & 65.6 & 2.6 & 69.1 & 2.3 \\
\hline 1890 & 90.7 & 2.4 & 91.3 & 2.2 & 92.1 & 2.0 & 93.2 & 1.7 \\
\hline 1902 & 26.9 & 1.9 & 25.1 & 1.8 & 22.0 & 1.6 & 18.3 & 1.4 \\
\hline 1907 & 75.8 & 1.9 & 74.2 & 2.2 & 76.1 & 2.0 & 78.9 & 1.6 \\
\hline 1919 & 84.0 & 1.9 & 83.4 & 2.1 & 83.4 & 2.0 & 83.6 & 1.8 \\
\hline 1932 & 69.0 & 1.8 & 69.9 & 1.9 & 68.2 & 1.7 & 65.3 & 1.6 \\
\hline 1939 & 96.3 & 2.1 & 96.2 & 2.1 & 96.3 & 2.0 & 96.5 & 1.8 \\
\hline 1946 & 94.9 & 0.6 & 94.9 & 0.6 & 95.1 & 0.6 & 95.3 & 0.6 \\
\hline 1955 & 97.7 & 1.9 & 97.6 & 2 & 97.7 & 1.8 & 97.9 & 1.6 \\
\hline 1962 & 30.8 & 1.1 & 23.8 & 1.3 & 28.5 & 1.3 & 35.1 & 1.2 \\
\hline & 68.7 & 1.9 & 66.2 & 2.0 & 67.2 & 1.8 & 68.6 & 1.6 \\
\hline
\end{tabular}

Table 7. Correlation [\%] and RMS [cm] of reconstructed HIS signal to the original ESA ESM HIS for the 15 selected study cases with a moving average applied (d/o 30, circles $R=4^{\circ}$ ).

\begin{tabular}{|c|c|c|c|c|c|c|c|c|}
\hline \multirow[t]{3}{*}{ ID } & \multicolumn{8}{|c|}{ Reconstructed HIS (AO + Error) vs. H } \\
\hline & \multicolumn{2}{|c|}{ Circle, $R=4^{\circ}$} & \multicolumn{2}{|c|}{ MovAv., $d=31$} & \multicolumn{2}{|c|}{ MovAv., $d=15$} & \multicolumn{2}{|c|}{ MovAv., $d=7$} \\
\hline & Corr [\%] & RMS [cm] & Corr [\%] & RMS [cm] & Corr [\%] & $\mathrm{RMS}[\mathrm{cm}]$ & Corr [\%] & RMS [cm] \\
\hline 1863 & 47.5 & 1.6 & 46.8 & 1.0 & 46.2 & 0.8 & 46.1 & 0.8 \\
\hline 1866 & 42.7 & 1.3 & 43.4 & 0.7 & 42.7 & 0.5 & 36.8 & 0.5 \\
\hline 1870 & 16.8 & 1.7 & 19.8 & 0.9 & 18.0 & 0.6 & 16.9 & 0.4 \\
\hline 1881 & 38.2 & 1.8 & 39.9 & 1.0 & 40.4 & 0.8 & 40.3 & 0.9 \\
\hline 1885 & 90.0 & 2.2 & 90.0 & 1.5 & 90.1 & 1.5 & 90.5 & 2.0 \\
\hline 1886 & 49.0 & 2.3 & 49.2 & 1.5 & 49.6 & 1.3 & 51.1 & 1.3 \\
\hline 1890 & 62.1 & 1.8 & 61.8 & 1.2 & 61.0 & 1.1 & 60.7 & 1.2 \\
\hline 1902 & 12.4 & 1.5 & 12.3 & 1.2 & 13.7 & 1.1 & 13.1 & 1.0 \\
\hline 1907 & 49.5 & 1.7 & 50.2 & 1.0 & 50.4 & 0.8 & 50.6 & 0.7 \\
\hline 1919 & 71.0 & 2.4 & 71.1 & 1.9 & 71.8 & 1.8 & 71.6 & 1.8 \\
\hline 1932 & 53.4 & 1.6 & 54.2 & 1.1 & 53.9 & 0.9 & 52.6 & 0.8 \\
\hline 1939 & 93.5 & 2.3 & 93.4 & 1.8 & 93.2 & 1.8 & 93.6 & 1.8 \\
\hline 1946 & 79.9 & 3.4 & 80.6 & 3.4 & 80.6 & 3.4 & 81.2 & 3.4 \\
\hline 1955 & 84.7 & 1.8 & 84.5 & 1.3 & 84.6 & 1.3 & 85.3 & 1.6 \\
\hline \multirow[t]{2}{*}{1962} & 44.3 & 1.1 & 43.1 & 0.8 & 42.6 & 0.5 & 42.6 & 0.4 \\
\hline & 55.7 & 1.9 & 56.0 & 1.4 & 55.9 & 1.2 & 55.5 & 1.2 \\
\hline
\end{tabular}

